\title{
A New Approach to Design Light Emitting Devices Using Electroactive Dyes
}

\author{
Michael Pan, Amitava Patra, Christopher S. Friend, Tzu-Chau. Lin, Alexander N. \\ Cartwright, and Paras. N. Prasad \\ Institute for Lasers, Photonics and Biophotonics, Departments of Electrical Engineering and \\ Chemistry, University at Buffalo, The State University of New York, Buffalo, NY 14260 \\ Ryszard Burzynski \\ Laser Photonics Technology, Inc., 1576 Sweet Home Road, Amherst, New York 14228
}

\begin{abstract}
Organic electroluminescence (EL) single layer devices using electroactive dyes incorporated in poly-vinylcarbazole (PVK) were fabricated. The molecular structures of the twophoton dyes are the generic $D-\pi-A, D-\pi-D$, and $A-\pi-A$, structural motif, composed of a diphenylamine donor (D), a distyrylfluorene $\pi$-bridge, and an oxadiazole acceptor (A). A single layer type of EL device of ITO/PVK:DYE/Ca/Al was fabricated. The light emission peak and the threshold of the electroluminescence emission depend on the structure and concentration of the dye. The EL intensity increases with the dye concentration and it was found that as the voltage is increased the brightness increases and reaches a value $498 \mathrm{~cd} / \mathrm{m}^{2}$ at an applied voltage of $25 \mathrm{~V}$ for the D- $\pi-A$ dye. We present a physical explanation of this observed behavior and show that this has significant impact on the design of light emitting devices using these organic dyes.
\end{abstract}

\section{Introduction}

Since the first demonstration of a green organic light emitting diode in 1987 by Tang and VanSlyke [1], numerous organic molecules and polymers have been found that exhibit electroluminescence in the blue, green and red spectral region [2,3]. Organic electroluminescent devices represent a low cost route for display technology. That is, for development of large area light-emitting displays there is considerable interest in the study of electroluminescent polymers because of the structural flexibility, low cost of fabrication and low operation voltage. A variety of organic materials including polymers [1- 6], metal complexes [7] and fluorescent dyes [8,9] have demonstrated electroluminescence since organic materials generally produce emission due to the $\pi-\pi *$ transition. In fact, the generation of light in the device is due to recombination of electrons and holes injected from the electrodes. The efficiency of a LED depends on the choice of the molecules and on the design of the device structure. Generally, layered devices exhibit better performance than a single layer device because the optimization of injection and recombination of holes and electrons is simplified [6]. Furthermore, the efficiency of the electroluminescent organic light-emitting device can be improved by introduction of a phosphorescent sensitizer to excite fluorescent dyes [10]. The efficiency of electron-hole recombination leading to the creation of singlet excitons is mainly influenced by the overlap of electron and hole densities that originate from carrier injection into the emitter layer [11]. To improve the efficiency of carrier injection organic hole and electron injection layers with a low HOMO (highest occupied molecular orbit) or high LUMO (lowest unoccupied molecular orbital) level are chosen respectively. Therefore, the structure of the molecule plays an important role to develop an efficient device. 
Two-photon dyes are well-known for use in nonlinear applications because of their high two-photon absorption cross-section [12]. From the viewpoint of the molecular structure, the two-photon dye (PRL-801) used in this work has a generic D- $\pi$-A structural composed of a diphenylamine donor (D), a distyrylfluorene $\pi$-bridge, and an oxadiazole acceptor (A). These types of molecules are called "push-pull" type chromophores owing to their asymmetrically substituted nature. Despite the original design consideration of this chromophore for NLO studies, one can also see this molecule in a different way. In fact, it is worth mentioning that several features from the substructures or building blocks of this chromophore may affect the electronic and emission properties of the whole molecule to fit the basic requirement of organic light emitting diode. In order to investigate the molecular structural effect on the electroluminescence property, we also incorporated two additional chromophores structures bearing either two electron donors (triphenylamine) designated as PRL-803 or two electronacceptors (oxadiazole) designated as PRL-802 for comparison. These structures are symmetrically substituted in the form of $D-\pi-D$ or $A-\pi-A$ type. The structures of these two dyes are shown in figure 1. In this work we demonstrate a single layer EL device using two-photon dyes which may lead to new applications for these dyes.

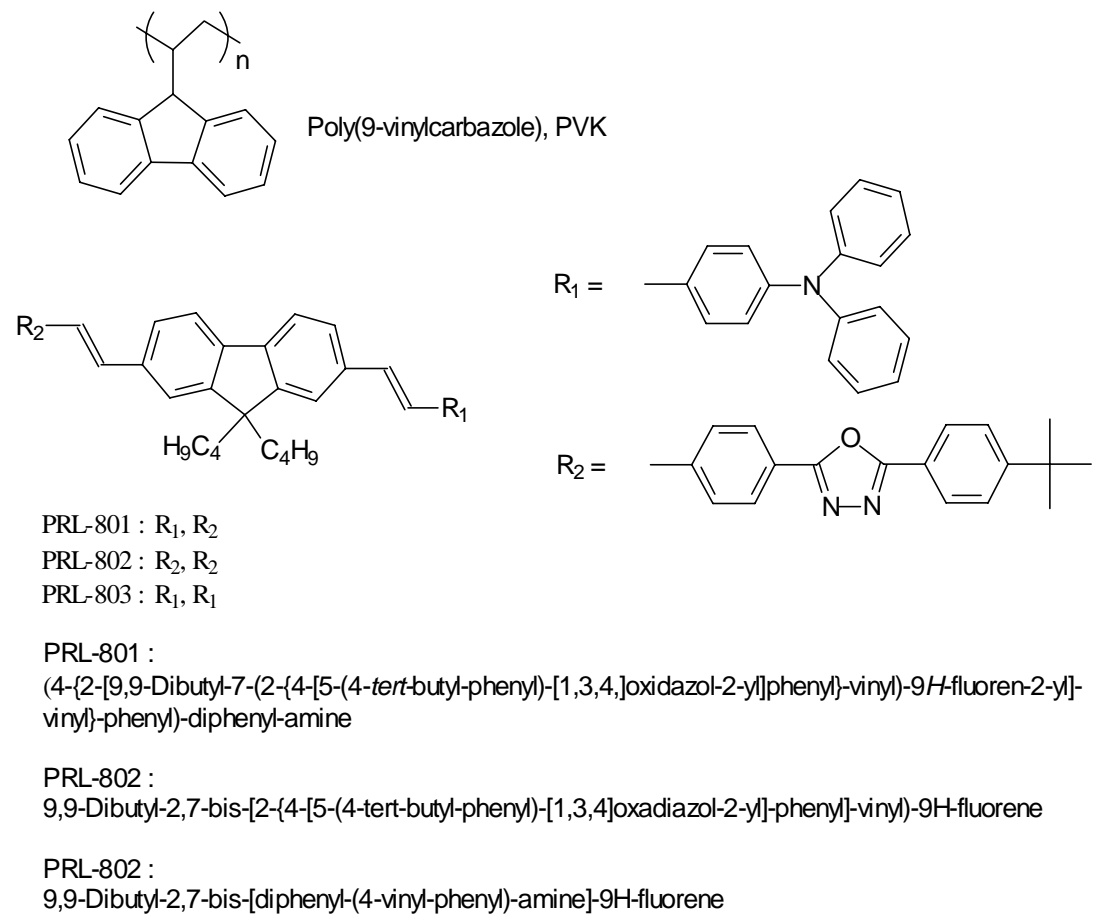

Figure 1. The molecular structure of the dyes used in this work.

\section{Experimental}

To fabricate EL devices poly(N-vinylcarbazole) PVK was employed as a host polymer. A carbazole group in PVK is well-known for transporting holes and blocking the electrons. The thin films of PVK doped with dye were prepared by spin coating from a PVK:DYE (with different concentrations) chloroform solution. Indium-tin-oxide coated glass (ITO) substrates with a sheet resistance of $20 \Omega$ /square were etched to form strips $2 \mathrm{~mm}$ wide to be used as the 
device anode. The thickness of the PVK-DYE film was about $140 \mathrm{~nm}$. A single layer type of EL device consisting of ITO/PVK:DYE/Ca/Al was constructed. The calcium and aluminum were thermally deposited in a Denton high vacuum chamber at $6 \times 10^{-6}$ torr. The optimal electrode thickness for calcium was determined to be 100 nanometers, and the aluminum thickness was observed to have very little or no effect on the optimization of the device efficiency. The aluminum was deposited as a protective layer. The luminescent areas for the devices are fairly large and were set at $7.9 \mathrm{~mm}^{2}$ for the small cell, and $18 \mathrm{~mm}^{2}$ for the large cell area of the circular electrode. The turn on voltage varies with concentration and depends upon the specific dye used. Both photoluminescence (PL) and electroluminscence (EL) were measured at room temperature and atmospheric pressure. The samples were not hermetically sealed from the atmosphere. Electroluminescence was measured in continuous DC mode. The I-V characteristics were recorded using a Keithley 208 high current source measurement unit. The absolute luminescence intensity was measured with a Minolta LS-110 Luminance meter. For the time-resolved PL measurements, the $800 \mathrm{~nm}, 150$ fs laser pulses from a $250 \mathrm{kHz}$ regenerative amplifier (REGA) were frequency doubled to $400 \mathrm{~nm}$ to serve as the pump source. The resulting photoluminescence was spectrally and temporally resolved using a Hamamatsu streak camera with 20 ps response time.

\section{Results and Discussion}

The photoluminescence (PL) and the electroluminescence (EL) spectra of the single layer structure of PRL-801 with different concentrations are shown in figure 2.

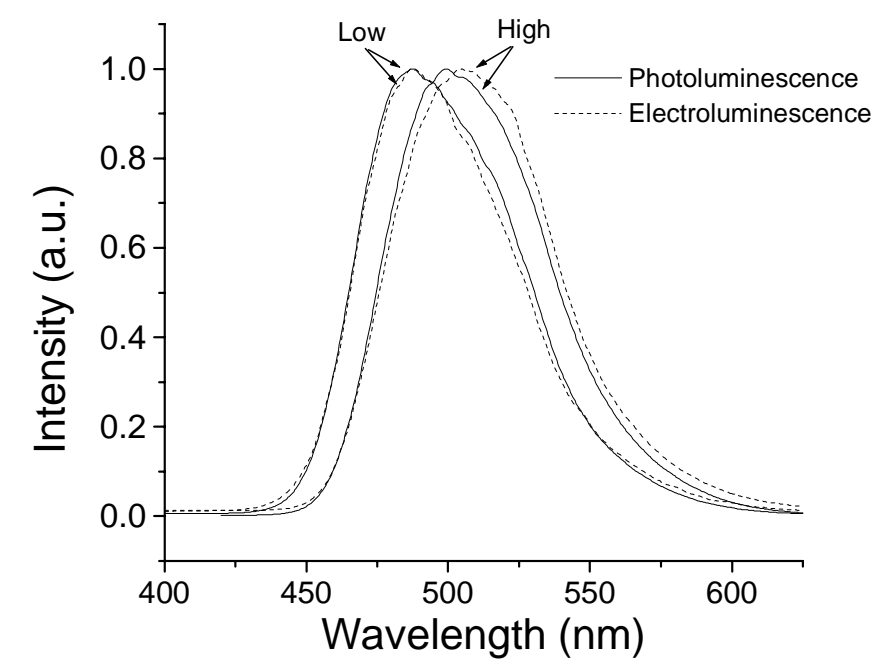

Figure 2. The photoluminescence (PL) and the electroluminescence (EL) spectra of the single layer structure of PRL-801 with different concentrations (28.5 wt \%, high; $4.76 \mathrm{wt} \%$, low).

The PL peak shifted towards longer wavelength with increasing concentration as expected since excimer states form at high concentrations [13]. Moreover, we observed an increase in EL intensity and a reduction in turn-on voltage with increasing concentration of the dye. Specifically, the EL intensities are 111.6 and $3.01 \mathrm{~cd} / \mathrm{m}^{2}$ for high and low at an applied voltage of 20 volts. The turn-on voltages are 9 and 14 volts for high and low. Similar observations where 
the EL efficiency depends much more on dye concentration than the PL efficiency have already been reported [14].

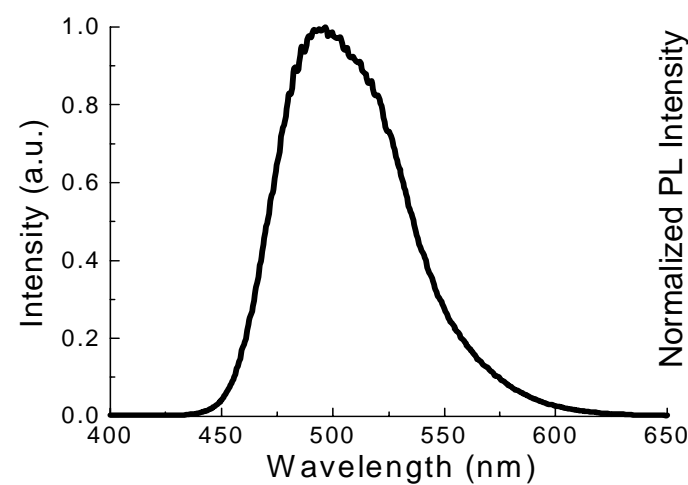

(a)

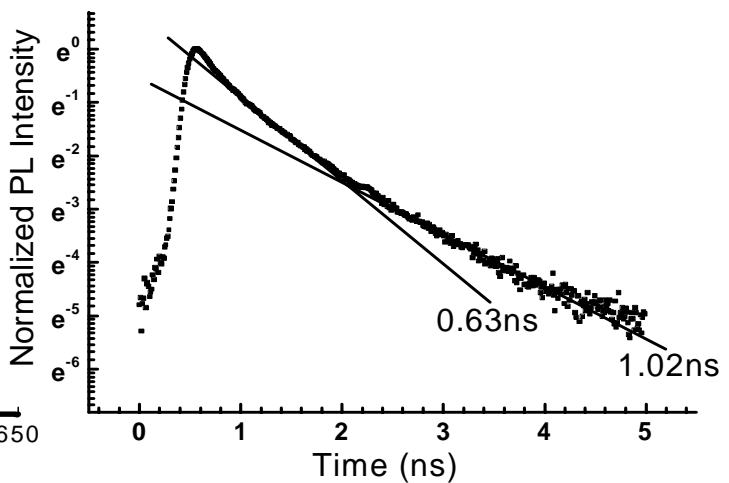

(b)

Figure 3. Photoluminescence spectra (a) and temporal decay of the photoluminescence (b) containing PRL-801 dye (16.6 wt \%).

Figure 3a shows the photoluminescence spectra of PRL-801 dye (16.66 wt \%). Figure 3b shows the photoluminescence decay of PRL-801 dye (16.66 wt \%) at peak emission wavelength. The overall photoluminescence decay with an excitation at $400 \mathrm{~nm}$ at peak PL emission shows

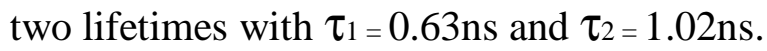

As stated earlier, the ITO anode has a relatively high work function and is a good material for injecting holes. Calcium and aluminum provided the best combination for the cathode material in this study. Figure 4 presents an energy level diagram for the devices prepared in this work. Calcium was chosen because of its low work function $(2.87 \mathrm{eV})$, and thus the barrier for electron injection from the metal cathode to LUMO of the emitting layer is small. Injection of electrons into the device is more efficient with $\mathrm{Ca} / \mathrm{Al}$ compared with only $\mathrm{Al}$. In most organic LEDs reported so far, the EL originated from singlet excited states of the fluorescent dyes or polymers used as the emitting materials. Ionization potentials, Ip, and

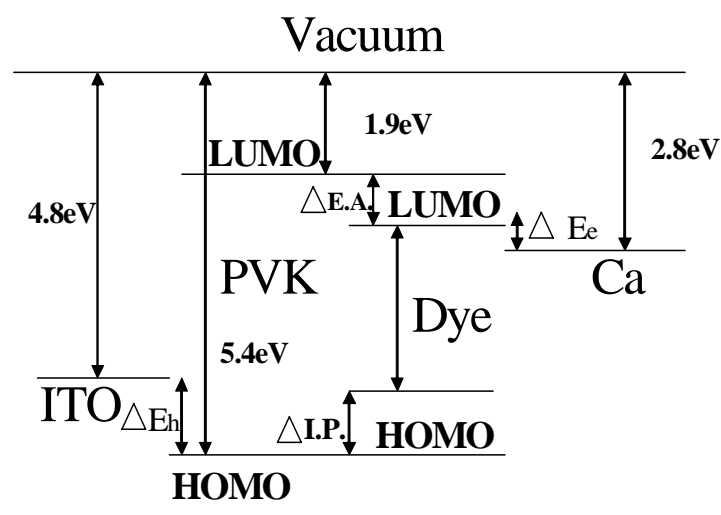

Figure 4. Schematic energy level diagram of the Light Emitting Diode (ITO/PVK$\mathrm{DYE} / \mathrm{Ca} / \mathrm{Al})$. electron affinities, Ea, are not yet available for our dye.

When a bias is applied to the device, holes are injected into the HOMO of the carbazole side of PVK and the electrons are injected into the LUMO of oxadiazole part of the dye (PRL-801), which is an electron-transporting media and charge carrier recombination generates the singlet excited states of the dye. Because in the electron transfer process, electron transfer occurs between the LUMO's of the donor and acceptor and in case of hole transfer process, the hole transfer occurs between the HOMO's. It is expected that the efficiency of electron-hole recombination is influenced by the overlap of electron and hole 
densities that originate from carrier injection into the dye molecule.
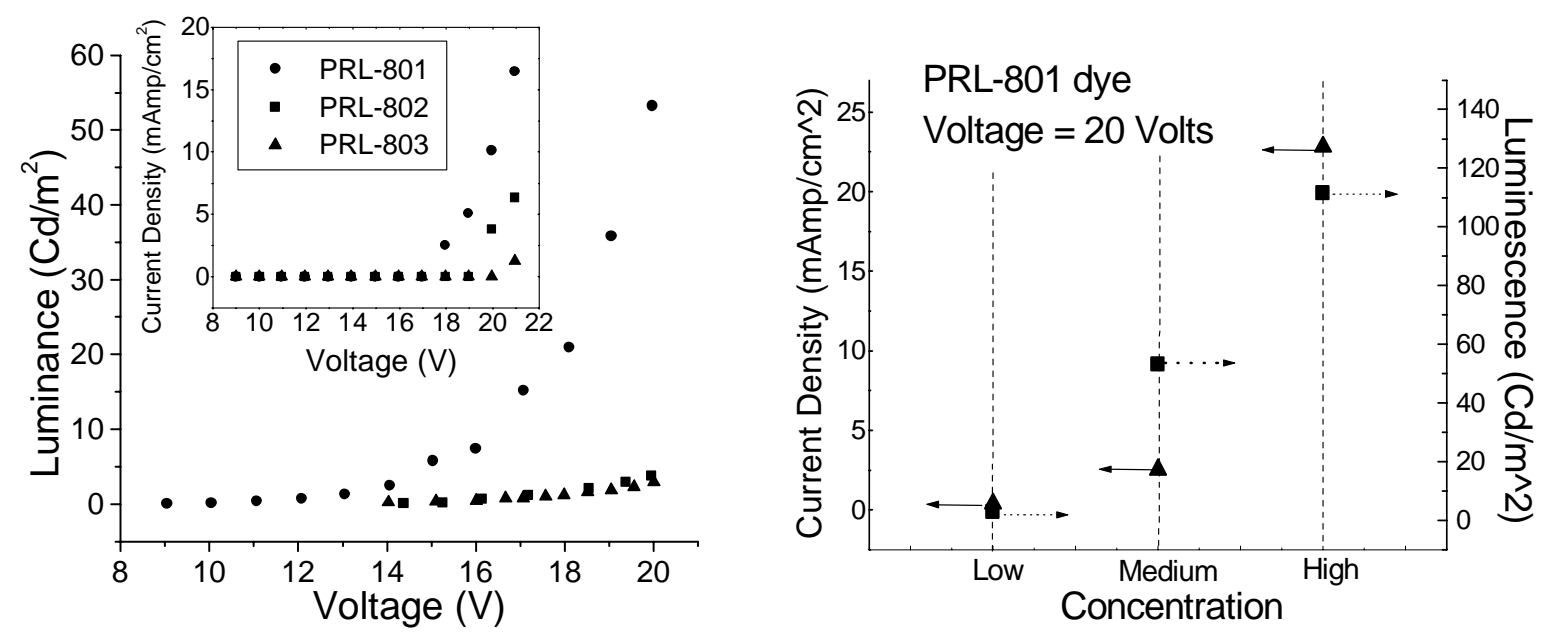

Figure 4. (a) The I-V and EL-V characteristics of the single layer OLED structure containing PRL-801, PRL-802 or PRL-803 dye at constant concentration of $16.66 \mathrm{wt} \%$ and (b) the Current Density-Concentration and EL-Concentration characteristics of the single layer structure of PRL-801 at different concentrations (28.5 wt $\%$, high; $16.6 \mathrm{wt} \%$, medium; $4.76 \mathrm{wt} \%$, low).

Figure 4(a) shows the I-V and EL-V characteristics of the single layer structure of PRL801, PRL-802 and PRL-803 having the same dye concentration (16.6 wt \%) and the EL intensities are $35.85,2.94,1.87 \mathrm{~cd} / \mathrm{m}^{2}$ at $19 \mathrm{~V}$, which correspond to emitted electroluminescence powers of 0.06433 and 0.0094 and $0.0045 \mu \mathrm{W}$ respectively. The threshold of the electroluminescence emission depends on the structure and concentration of the dye. The light was clearly visible under room light. As the voltage is increased both the injection current and the luminescence intensity increase in an exponential manner. Figure 4(b) shows Current DensityConcentration and EL-Concentration characteristics of the single layer structure of PRL-801 at an applied voltage of $20 \mathrm{~V}$ (28.5 wt $\%$, high; $16.6 \mathrm{wt} \%$, medium; $4.76 \mathrm{wt} \%$, low). The

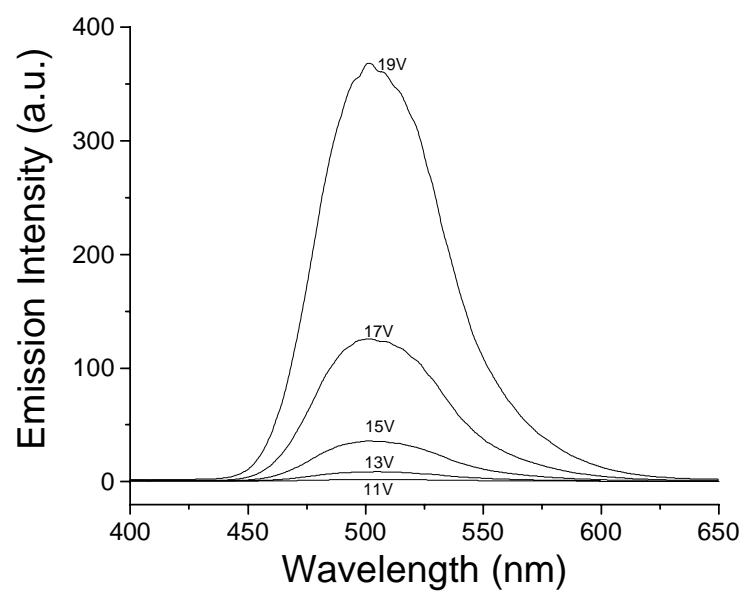

Figure 6. Electroluminescence (EL) of the device of PRL-801 (16.66 wt \%) with various operating voltage. electroluminescence intensities are 111.6, 53.24 , and $3.01 \mathrm{~cd} / \mathrm{m}^{2}$ and current density are 22.8, 2.53, $0.4 \mathrm{mAmp} / \mathrm{cm}^{2}$ for high, medium, and low respectively.

Figure 6 shows the electroluminescence spectra at different applied voltages at room temperature. As the voltage is increased the brightness also increases and reaches a value of $498 \mathrm{~cd} / \mathrm{m}^{2}$ at an applied voltage of $25 \mathrm{~V}$ for PRL-801 dye. However, further experiments are needed to increase the lifetime and stability of these devices. The PRL-801 dye doped device is the most efficient among the two-photon dyes. The better performance of the device based on PRL-801 is thought to be 
due to the better balance in the number of injected holes and electrons compared to the other dyes because this dye contains both the donor and acceptor groups.

\section{Conclusion}

Our results show that the new electroactive molecule having both electron- transporting and hole-transporting groups in their structure produce efficient EL emission in a single layer device without using separate hole and electron transfer layers. It is expected that the efficiency of this device can be improved by changing the molecular structure of the dye, i.e., varying the electron-donating or electron-accepting strength of the end groups, introducing additional groups in the middle to vary the charge distribution, and varying the effective conjugation length in order to achieve design criteria to produce structures with enhanced electroluminescence efficiency.

\section{References}

1. C. W. Tang and S. A. Vanslyke, Appl. Phys. Lett., 51, 913 (1987).

2. S. Saito, T. Tsutsui, M. Era, N. Takada, C. Adachi, Proc. SPIE, 1910, 212 (1993).

3. D. R. Baigent, N. C. Greenham, J. Gruner, R. N. Marks, R. H. Friend, S. C. Moratti, A. B. Holmes, Synth. Met., 67, 3 (1994).

4. J. H. Burroughes, D. D. C. Bradley, A. R. Brown, R. N. Marks, K. Mackay, R. H. Friend, P.L. Bruns and A. B. Holmes, Nature (London) 374, 539 (1990).

5. D. Braun and A. J. Heeger, Appl. Phys. Lett, 58, 991 (1982).

6. D.D. Gebler, Y.Z. Wang, J.W. Blatchford, S.W.Jessen, D. K. Fu, T. M. Swager A.G. MacDiarmid and A. J. Epstein, Appl.Phys.Lett., 70, 1644 (1997).

7. T. Sano, M. Fujita, T. Fujii, Y. Hamada, K. Shibata and K. Kuroki, Jpn. J. Appl. Phys. 34, 1883 (1995).

8. J. Kido, M. Kohda, K. Okuyama and K. Nagai, Appl. Phys. Lett., 61, 761 (1992).

9. H. Vestweber, R. Sander, A. Greiner, W. Heitz, R. F. Mahrt and H. Bassler, Synth. Met. 64, 141 (1994).

10. M. A. Baldo, D. F. O’Brien, Y. You, A. Shoustikov, S. Sibley, M. E. Thompson and S. R. Forrest, Nature, 395, 151 (1998).

11. K. C. Kao, W. H. Wang, Electrical transport in solids, Pergamon Press, Oxford, 1981.

12. S.J. Chaung, K.S. Kim, T.C.Lin, G.S. He, J. Swiatkiewicz and P. N. Prasad, J. Phys. Chem. B, 103, 1074 (1999).

13. M. Pope and C. E. Swenberg, Electronic Processes in Organic Crystals (Oxford University Press, New York, 1982).

14. F. Pschenitzka and J. C. Sturm, Appl. Phys. Lett., 79, 4354 (2001).

15. J. Thompson, R. I. R. Blyth, M. Mazzeo, M. Anni, G. Gigli and R. Cingolani, Appl. Phys. Lett., 79, 560 (2001).

16. C.I.Chao and S.A. Chen, Appl. Phys. Lett., 73, 426 (1998). 